\title{
Stress computations on perforated polygonal domains
}

\author{
Jonas Englund, Johan Helsing* \\ Numerical Analysis, Centre for Mathematical Sciences, Lund University, Box 118, Lund S-221 00, Sweden
}

Received 16 January 2002; revised 14 June 2002; accepted 28 June 2002

\begin{abstract}
A high order accurate and fast algorithm is constructed for 2D stress problems on multiply connected finite domains. The algorithm is based on a Fredholm integral equation of the second kind with non-singular operators. The unknown quantity is the limit of an analytic function. On polygonal domains there is a trade-off between stability and rate of convergence. A moderate amount of precomputation in higher precision arithmetic increases the stability in difficult situations. Results for a loaded single edge notched specimen perforated with 1170 holes are presented. The general usefulness of integral equation methods is discussed.

(C) 2003 Elsevier Science Ltd. All rights reserved.
\end{abstract}

Keywords: Multiply connected domain; V-notch; Holes; Notch stress intensity factor; Stress concentration factor; Fredholm integral equation; Fast multipole method

\section{Introduction}

The problem of solving for stress in a loaded, linearly elastic body has received attention for almost a century. Early work dealt with formulations of the governing equations. Integral equations appeared as a natural tool both for analysis and for obtaining solutions. See the textbooks of Mikhlin [40], Muskhelishvili [41] and Sokolnikoff [48]. Naturally, only very simple setups can be solved by purely analytical methods.

Later work on stress problems has focused more on numerical techniques for particular applications. See Refs. $[7,9,10,14,30,38,50,52]$ for recent $2 \mathrm{D}$ examples involving a variety of methods of which some are based on integral equations. Parallel to this development there has been a steady improvement of commercial general purpose finite element packages, such as ANSYS and ABAQUS. These codes can, among other things, solve for stress on multiply connected 2D domains. In fact, in Ref. [29] we showed that for simple setups, involving a few holes and cracks in finite domains, these commercial packages often perform better than many specialized codes. Similar observations made by others has lead to a belief in the solid mechanics community that the finite element method will be the only numerical

\footnotetext{
* Corresponding author. Tel.: + 46-46-2223372; fax: +46-46-2220595.

E-mail address: helsing@maths.lth.se (J. Helsing).
}

method in the future. Once computer processors become fast enough, and computer memories become large enough, the need for other numerical techniques will disappear.

In this paper we argue and demonstrate that integral equation methods are indeed extremely competitive, that is, when they apply and if they are implemented carefully. We ascribe their limited use in computational solid mechanics to insufficient work on good equations for problems of interest. The paper is organized as follows: Section 2 discusses advantages with and shortcomings of integral equation methods. Section 3 reviews a recently derived and very efficient equation for multiply connected $2 \mathrm{D}$ domains [29]. Sections 4 and 5 contain the new material. Section 4 gives details on the present discretization of the integral equation. Particular emphasis is given to the quadrature in corners. Our scheme for resolving the stress field in corners, originally developed in Ref. [28], makes use of the analytic properties of the unknown quantity, and should be seen as an alternative to the more general scheme of Cheng, Yarvin and Rokhlin [12,53]. New placement of discretization points increases the stability. Higher order quadrature increases the convergence rate, compared to the earlier implementation $[28,29]$. Section 5 gives several numerical examples. Among other things, we compute the notch stress intensity factor and the stress concentration factor for a loaded single edge notched specimen perforated with 1170 circular holes. 


\section{Why are integral equation methods needed?}

The chief advantage of integral equation methods over methods based on partial differential equations has to do with stability. With stability, in this context, we mean that the truncation error should decrease when the number of discretization points is increased while the rounding error should stay bounded. The rounding error is not bounded for methods that, upon discretization, lead to system matrices whose condition numbers diverge with the number of discretization points.

Since many problems in linear fracture mechanics are well conditioned [39], a good numerical method for these problems should result in discretized problems with low condition numbers. Algorithms based on partial differential equation formulations do not have this property. Differential operators are unbounded and when the discretization is refined, the rounding error increases. These undesirable effects could, in theory, be offset by preconditioners using techniques such as multigrid [24] or domain decomposition [4]. Still, for many realistic problems and geometries it is often difficult to achieve optimal performance [15]. The total work tends to grow faster than linearly with the number of discretization points $N$. The achievable accuracy decreases as the system size increases.

When a given problem can be formulated as a Fredholm integral equation of the second kind it may happen that its condition number is preserved. And even if the condition number is not exactly preserved, it does not depend on the number of discretization points (see Theorem 4.1.2 of Ref. [3] and Theorem 4.7.11 of Ref. [25]). Iterative schemes then converge rapidly and there is no need for preconditioners. The computing time grows linearly with $N$, given that fast solvers are used. The accuracy can be controlled. It should be pointed out that the preservation of condition number does not automatically follow from a Fredholm equation formulation. Neither does it exist a general recipe for the derivation of integral equations with this property. But for an increasing number of problems in science and engineering, usually formulated as partial differential equations, it has been possible to find reformulations where the condition number is almost preserved.

Despite the advantages mentioned above, integral equation methods are not standard for solving field problems. There are several reasons for this. One is historical. It was not until about 15 years ago that fast iterative solvers for integral equations in 2D first appeared $[5,20,45]$. Efficient codes for 3D have been finalized in the last few years [13,21]. Another reason, particularly relevant for solid mechanics, has to do with the difficulties of finding good integral equations for problems of interest. Sometimes, for simple geometries and linear problems, there are several equations to choose between, many of which were developed a long time ago and without numerical applications in mind. Comparative studies of the performance of these equations seem to be lacking. Often, for more complicated geometries, for problems with continuously varying material coefficients, and for non-linear problems, there are no equations available at all. Researchers working with efficient algorithms for large scale-problems do not always address these questions. They often concentrate on the speed of their solvers and then apply their algorithms to idealized problems. See, for example, Refs. [16,18,19,26, 54].

In other areas of scientific computing, such as scattering problems, integral equation methods have had much more of a revival [22]. Here their advantages over other methods are more easily exploited. Integral equations for problems of interest are available.

Other advantages with integral equations, often mentioned, but in our opinion less important than the stability properties, have to do with economy of points and ease of mesh-generation. For free-space problems and for problems involving piecewise homogeneous materials, integral equation methods often only require the discretization of the boundary.

It is clear, from above, that the present authors prefer to work with Fredholm integral equations of the second kind for stability reasons. It should be emphasized that most contemporary researchers working with integral equation solvers in solid mechanics use other approaches. The reason is that integral equations of the second kind are often very hard to find for a given problem. If one extends one's search to Fredholm equations of the first kind it is easier to find candidates. In fact, many popular integral equation used for fracture mechanics problems $[8,37,44]$ (hypersingular extensions of the Somigliana identity [34], also known as standard BEM) are equations of this type. Schemes based on first kind equations retain some of the advantages of second kind integral equation schemes, such as ease of meshgeneration. Others are lost. Hypersingularity, which appears when double layer potentials are differentiated in the dual boundary boundary element method $[8,44]$, is in some sense similar to differentiation-compare the Cauchy integral formula for an analytic function $f(z)$ and $z$ inside $\Gamma$

$\frac{1}{2 \pi \mathrm{i}} \int_{\Gamma} \frac{f(\tau) \mathrm{d} \tau}{(\tau-z)^{2}}=f^{\prime}(z)$.

The properties of the hypersingular integral equation resemble those of a differential equation. In general, the condition number of system matrices that results from first kind equations have condition numbers that grow like $\mathrm{O}(N)$ [6]. For simple problems, solved with direct solvers, this is not a worry. For difficult problems and when iterative solvers are needed, the high condition number might negatively affect the accuracy of the solution and it may lead to unreasonably long execution times.

Because of the unbounded condition numbers for matrices of discretized first kind equations, preconditioning might be needed. There are several approaches for integral equations. Multigrid type preconditioners [43] and additive Schwarz preconditioners [1] are two examples which have 
successfully been used for finite element equations. A preconditioning technique that is not an analogue to an existing finite element preconditioner, but takes advantage of the inherent properties of the integral equation, can be found in Steinbach and Wendland [49]. Here a discrete boundary integral operator of order $-m$ is used to precondition a boundary integral operator of order $m$. The idea is to mimic analytic left regularization. Time will tell if schemes using preconditioned integral equations will be competitive to differential equation based schemes such as the finite element method. The experience with solving truly large-scale problems is still very minimal.

In summary, numerical methods based on integral equations of Fredholm's second kind can be extremely stable. Their advantages over methods based on partial differential equations are most pronounced for large and illconditioned problems. Therefore, we argue, unless even more efficient numerical methods emerge [15], integral equation methods will become increasingly appreciated as computers get more powerful and more difficult problems are attacked. New integral equations need to be developed for solid mechanics problems.

\section{Multiply connected finite 2D domains}

In this section we review an integral equation for stress problems on multiply connected finite $2 \mathrm{D}$ domains. There are different opinions about what integral equation to use for this problem. Classic authors [40,41,48] recommend an approach starting with the Airy stress function and the Sherman-Lauricella representation. Another option is to use 'standard BEM', derived from the Kelvin fundamental solution to the elastostatic partial differential equation. Both these approaches, involving logarithmic kernels, have been reported to generate system matrices whose condition numbers grow with the system size [19,35]. Preconditioners are needed. We instead use a recently derived [29] nonsingular Fredholm second kind equation which is free from this problem. Furthermore, the unknown quantity $\Omega(z)$ in the integral equation is the limit of an analytic function. This enables the use of high-order quadrature in the solution process also for polygonal domains.

\subsection{The integral equation}

A finite, linearly elastic, specimen occupies a domain $D$ in the complex plane. The outer boundary of the specimen is denoted $\Gamma_{0}$ and is given positive (counter-clockwise) orientation. The domain $D$ is multiply connected. Inside $D$ there are a number $N_{\mathrm{h}}$ of holes. The holes have boundaries $\Gamma_{j}, j=1,2, \ldots, N_{\mathrm{h}}$, and are given positive orientations. The union of all boundaries is $\Gamma$. The area of the region enclosed by contour $\Gamma_{j}$ is $A_{j}$. Traction $t^{\mathrm{pr}}=t_{x}^{\mathrm{pr}}+\mathrm{i} t_{y}^{\mathrm{pr}}$, that is, stress on the boundary, is prescribed at $\Gamma_{0}$. The holes are free of stress. The exterior of the domain is denoted $D^{\prime}$.
Our integral equation [29] reads

$\left(I-M_{3}-2 \mathrm{i} P_{0}\right) \Omega(z)=\frac{1}{2}\left(I-\frac{\bar{n}}{n} \bar{M}_{1} \frac{n}{\bar{n}}\right) \bar{n} t^{\mathrm{pr}}$,

$z \in \Gamma_{0}$,

$\left(I+M_{3}+\frac{1}{2} P_{j} \mathrm{i}\left(I+M_{1}\right)+\mathrm{i} \bar{z} Q_{j}\right) \Omega(z)=\frac{1}{2} \frac{\bar{n}}{n} \bar{M}_{1} \frac{n}{\bar{n}} \bar{n} t^{\mathrm{pr}}$,

$z \in \Gamma_{j}, j=1,2, \ldots, N_{\mathrm{h}}$

Here $I$ is the identity and $z$ is a point in the complex plane. The operator $M_{3}$ can be regarded as compact, see Section 4.2. Its action on a function $f(z)$ is given by

$$
\begin{aligned}
M_{3} f(z)= & \frac{1}{2 \pi \mathrm{i}}\left[\int_{\Gamma} \frac{f(\tau) \mathrm{d} \tau}{(\tau-z)}+\frac{\bar{n}}{n} \int_{\Gamma} \frac{f(\tau) \mathrm{d} \tau}{(\bar{\tau}-\bar{z})}+\int_{\Gamma} \frac{\overline{f(\tau)} \mathrm{d} \bar{\tau}}{(\bar{\tau}-\bar{z})}\right. \\
& \left.+\frac{\bar{n}}{n} \int_{\Gamma} \frac{(\tau-z) \overline{f(\tau)} \mathrm{d} \bar{\tau}}{(\bar{\tau}-\bar{z})^{2}}\right], \quad z \in \Gamma
\end{aligned}
$$

where $n=n_{x}+\mathrm{i} n_{y}$ is the outward unit normal vector at $z$ on $\Gamma$. The operator $P_{j}$ is a mapping from $\Gamma_{j}$ to $\mathbb{R}$, defined by

$P_{j} f=-\frac{1}{2 A_{j}} \mathscr{R} e\left\{\int_{\Gamma_{j}} f(\tau) \bar{\tau} \mathrm{d} \tau\right\}$.

The Cauchy singular operator $M_{1}$, acting on a function $f(z)$, is given by

$M_{1} f(z)=\frac{1}{\pi \mathrm{i}} \int_{\Gamma} \frac{f(\tau) \mathrm{d} \tau}{(\tau-z)}, \quad z \in \Gamma$.

The conjugate of $M_{1}$ is denoted $\bar{M}_{1}$. The operator $Q_{j}$ is a mapping from $\Gamma_{j}$ to $\mathbb{C}$, defined by

$Q_{j} f=-\frac{1}{2 A_{j}} \int_{\Gamma_{j}} f(\tau) \mathrm{d} \tau$.

\subsection{Extraction of useful quantities from the solution}

Once Eqs. (1) and (2) are solved for $\Omega(z)$ several quantities of physical interest can be extracted. For example, the components of the stress tensor are

$\sigma_{x x}+\sigma_{y y}=4 \mathscr{R} e\{\Phi(z)\}$,

$\sigma_{y y}-\sigma_{x x}-2 \mathrm{i} \sigma_{x y}=2\left(z \overline{\Phi^{\prime}(z)}+\overline{\Psi(z)}\right)$,

where $\Phi(z)$ and $\Psi(z)$ are analytic functions in $D \cup D^{\prime}$ given by

$\Phi(z)=\frac{1}{2 \pi \mathrm{i}} \int_{\Gamma} \frac{\Omega(\tau) \mathrm{d} \tau}{(\tau-z)}, \quad z \in D \cup D^{\prime}$, 
and

$$
\begin{aligned}
\Psi(z)= & -\frac{1}{2 \pi \mathrm{i}} \int_{\Gamma} \frac{\overline{\Omega(\tau)} \mathrm{d} \bar{\tau}}{(\tau-z)}-\frac{1}{2 \pi \mathrm{i}} \int_{\Gamma} \frac{\bar{\tau} \Omega(\tau) \mathrm{d} \tau}{(\tau-z)^{2}} \\
& -\frac{1}{2 \pi \mathrm{i}} \int_{\Gamma_{0}} \frac{\bar{n} \overline{t^{\mathrm{pr}}} \mathrm{d} \tau}{(\tau-z)}, \quad z \in D \cup D^{\prime}
\end{aligned}
$$

A quantity of particular interest for perforated structures is the normalized tangential stress $\hat{\sigma}_{t}(z)$. One can define $\hat{\sigma}_{t}(z)$, for a stress-free hole, as the ratio of the trace of the stress tensor on the hole to the trace of the applied stress tensor. This quantity is easy to compute since $\Omega(z)$ is minus the outside limit of $\Phi(z)$ on the holes [29]. With external uniform unit traction applied in the $y$-direction we get

$\hat{\sigma}_{\mathrm{t}}(z)=-4 \mathscr{R} e\{\Omega(z)\}, \quad z \in \Gamma-\Gamma_{0}$.

A stress concentration factor $K_{\mathrm{t}}$ can be defined as the maximum absolute value of the normalized tangential stress

$K_{\mathrm{t}}=\max _{z \in \Gamma-\Gamma_{0}}\left|\hat{\sigma}_{\mathrm{t}}(z)\right|$

Should $\Gamma_{0}$ contain notches, it could be of interest to compute so-called notch stress intensity factors. The most common of these is the dimensionless mode I notch stress intensity factor $Q_{\mathrm{I}}$, defined in a Cartesian coordinate system, see Fig. 1, as

$Q_{\mathrm{I}} \equiv \lim _{x \rightarrow 0^{+}} \sqrt{2 \pi} \frac{\sigma_{y y}}{\sigma_{\text {ref }}}\left(\frac{x}{L}\right)^{1-\lambda_{1}}$,

where $L$ is a characteristic length scale, $\sigma_{\text {ref }}$ is a reference stress, and the limit is taken along the $x$-axis. For a single edge notched specimen under uniform uniaxial load, such as the one depicted in Fig. 2, a common choice is to take $L$ as the width $w$ of the specimen and $\sigma_{\text {ref }}$ as the magnitude of the prescribed traction. The exponent $\lambda_{1}$ in Eq. (13) is the first exponent in a local Williams series expansion [28,51] of the field $\Phi(z)$ in the notch (the re-entrant corner $E$ of Fig. 2)

$\Phi(z)=\sum_{n} f_{n} z_{*}^{\lambda_{n}-1}+\bar{f}_{n} z_{*}^{\bar{\lambda}_{n}-1}+g_{n} z_{*}^{\mu_{n}-1}-\bar{g}_{n} z_{*}^{\bar{\mu}_{n}-1}$,

where $f_{n}$ and $g_{n}$ are real or complex coefficients [28,51] and $z_{*}$ is the position in a local coordinate system with origin at the notch and oriented as in Fig. 1. The symmetric and

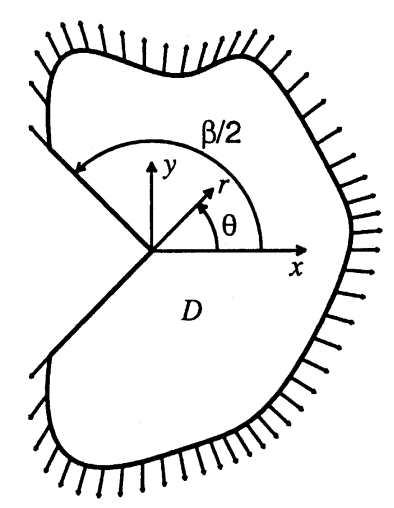

Fig. 1. A domain $D$ with a traction-free notch. The included vertex angle is $\beta$. The notch tip is at the origin. Traction is applied outside the notch.

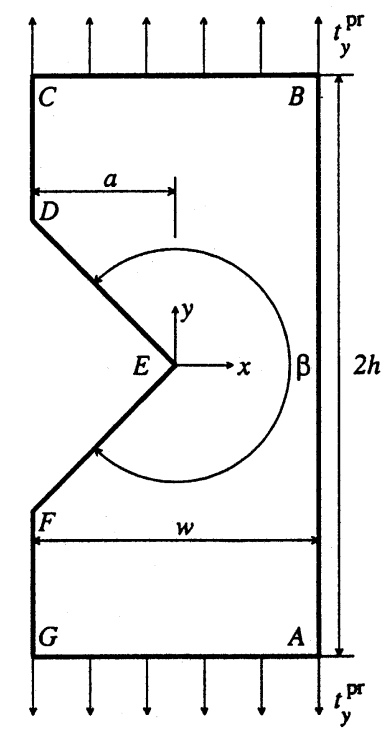

Fig. 2. A generic single edge notched specimen of length $2 h$ and width $w$. The notch depth is $a$. The included vertex angle is $\beta$. Uniform uniaxial traction $t_{y}^{\mathrm{pr}}$ is prescribed.

anti-symmetric exponents $\lambda_{n}$ and $\mu_{n}$ are given by the infinite number of solutions to the trigonometric equations

$\lambda \sin (\beta)+\sin (\lambda \beta)=0$,

$\mu \sin (\beta)-\sin (\mu \beta)=0$.

In Section 4, we discretize Eqs. (1) and (2) according to a Nyström scheme with composite quadrature. The corners will be symmetrically included in corner panels, each with two legs of length $l_{\mathrm{cp}}$. If the expansion of Eq. (14) is done in a local coordinate system where the corner legs have length unity, the notch stress intensity factor $Q_{\mathrm{I}}$ of Eq. (13) can be computed as

$Q_{\mathrm{I}}=\frac{f_{1}}{\sigma_{\text {ref }}} 2 \sqrt{2 \pi}\left(\lambda_{1}+1\right)\left(\frac{l_{\mathrm{cp}}}{w}\right)^{1-\lambda_{1}}\left[1-\frac{\cos \left(\left(\lambda_{1}-1\right) \beta / 2\right)}{\cos \left(\left(\lambda_{1}+1\right) \beta / 2\right)}\right]$.

There also exists a possibility to evaluate the notch stress intensity factor of Eq. (13) as a path-independent integral which encloses the notch [2,50]. An advantage with this post-processor is that it is less sensitive to the quality of the solution $\Omega(z)$ close to the notch than the formula (17). In fact, it can give reasonable estimates even if the solution at a few discretization points close to the notch-tip is completely wrong [50]. A drawback with the path-independent integral is that it is difficult to implement in an automated fashion. Since our scheme gives a rather accurate solution $\Omega(z)$ also close to the notch, see Section 4.4 , we prefer the much simpler formula (17).

\section{Implementation}

In this section we present details on our numerical algorithm for solving Eqs. (1) and (2). We try to be brief, 
yet detailed enough so that the general ideas will come through clearly.

\subsection{The mesh}

The integral equations (1) and (2) are to be discretized according to a Nyström scheme with composite quadrature. First, the boundary $\Gamma$ is divided into quadrature panels of approximately equal lengths. The panels are placed in such a way that whenever a panel contains a corner, the corner must be at the center of the panel. Corners containing panels are called corner panels. The two halves of the corner panels are called legs. The remaining panels are called regular panels. The solution $\Omega(z)$ will be singular on corner panels and smooth on regular panels. We intend to use polynomial quadrature on regular panels and a quadrature which takes the singular nature of the solution into account on corner panels. There is an extra need for resolution on regular panels neighboring to corner panels. Therefore these panels are subdivided twice. See Fig. 3 for an example where panels are placed on the boundary of the specimen in Fig. 2.

We intend to use Gauss-Legendre quadrature on the regular panels. The discretization points $z_{j}$ are placed accordingly. On the corner panels we intend to use a quadrature based on the expansion (14) and extra resolution of the kernels of $M_{1}$ and $M_{3}$, see Section 4.3. The number of discretization points for $\Omega(z)$ on a corner panel is related to how many terms that are used in expansion (14). The mapping from pointwise complex values of $\Omega(z)$ at the discretization points on a corner panel to the coefficients $f_{n}$ and $g_{n}$ preceding the basis functions $z_{* j}^{\lambda_{n}-1}, z_{* j}^{\lambda_{n}-1}, z_{* j}^{\mu_{n}-1}, z_{* j}^{\bar{\mu}_{n}}$ of Eq. (14) is given by inverting the relation

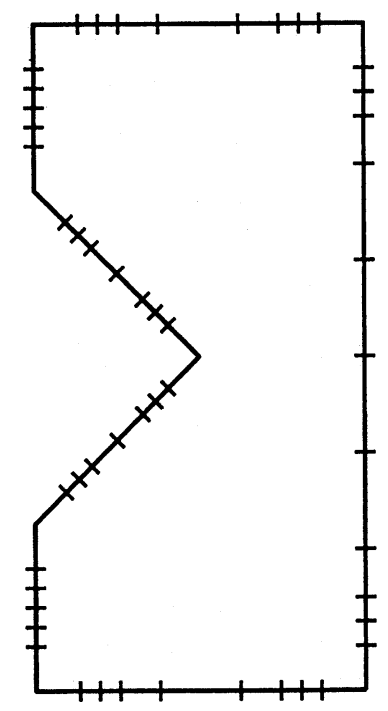

Fig. 3. The boundary of the single edge notched specimen of Fig. 2 is divided into quadrature panels. First, all panels are given approximately the same lengths. Then, panels neighboring to corner panels are subdivided twice.

$$
\begin{aligned}
& \Omega\left(z_{j}\right)= \sum_{n=1}^{N_{\mathrm{p}} / 2+m_{1}} \mathscr{R} e\left\{f_{n}\right\}\left(z_{* j}^{\lambda_{n}-1}+z_{* j}^{\bar{\lambda}_{n}}-1\right)+\sum_{n=1+m_{2}}^{N_{\mathrm{p}} / 2+m_{2}-m_{1}} \mathrm{i} \mathscr{I} m\left\{f_{n}\right\} \\
& \times\left(z_{* j}^{\lambda_{n}-1}-z_{* j}^{\bar{\lambda}_{n}-1}\right)+\sum_{n=1+m_{4}}^{N_{\mathrm{p}} / 2+m_{4}-m_{3}} \mathscr{R} e\left\{g_{n}\right\}\left(z_{* j}^{\mu_{n}-1}-z_{* j}^{\overline{\mu_{n}}-1}\right) \\
&+\sum_{n=1}^{N_{\mathrm{p}} / 2+m_{3}} \mathrm{i} \mathscr{I} m\left\{g_{n}\right\}\left(z_{* j}^{\mu_{n}-1}+z_{* j}^{\bar{\mu}_{n}-1}\right), \\
& j=1,2, \ldots, N_{\mathrm{p}},
\end{aligned}
$$

where $N_{\mathrm{p}}$ is the number of discretization points on the corner panel and the exponents $\lambda_{n}$ and $\mu_{n}$ are, for traction-free corners, the solutions to Eqs. (15) and (16). For corners that are not traction-free, but loaded with a uniform traction, the series $\lambda_{n}$ need to be completed with the exponent $\lambda=1$. The integers $m_{2}$ and $m_{4}$ in Eq. (18) denote the number of exponents $\lambda_{n}$ and $\mu_{n}$ which are real valued. The numbers $m_{1}$ and $m_{3}$ are chosen so that $2 m_{1} \approx m_{2}$ and $2 m_{3} \approx m_{4}$, that is, so that the series for real and imaginary parts of $f_{n}$ and $g_{n}$ are truncated after approximately the same number of terms. The number $N_{\mathrm{p}}$ will be chosen as an even number large enough so that the first omitted term in the series for $\lambda_{n}$ and $\mu_{n}$ is approximately equal for all corners. When the exponents $\lambda_{n}$ and $\mu_{n}$ lie widely spaced, we complete these series with integer exponents $1,2,3$, etc. so that we get at least $N_{\mathrm{p}}=8$ points on all corner panels.

\subsection{Avoiding singular operators}

Each of the four integrals appearing in the expression (3) for $M_{3}$ are singular and should be interpreted in Cauchy principal value sense. Fortunately, no Cauchy principal values need to be computed in the evaluation of $M_{3} \Omega(z)$. This is so since the sum of the kernels in the first and in the second integral of $M_{3}$ have the same (finite) left- and rightlimits as $\tau \rightarrow z$, at least for twice continuously differentiable curves, see also Remark 4.1. Similarly, the sum of the kernels in the third and in the fourth integral of $M_{3}$ have the same (finite) left- and right-limits as $\tau \rightarrow z$. No discretization points are placed in corners. Therefore, the kernel of the operator $M_{3}$ can be regarded as continuous and the operator is compact. The limiting values of the kernel, when $\tau=z$, are calculated analytically. The only problem with evaluating the kernel of $M_{3}$ occurs when $\tau$ is close to $z$. Then some cancellation will take place.

The kernel of $M_{1}$ in Eq. (5) is clearly singular if $z$ and $\tau$ are on the same quadrature panel. If $z$ and $\tau$ are on different panels the kernel is smooth and the operator is compact. The operator $M_{1}$ appears in three places in Eqs. (1) and (2) - twice on the right-hand side and once on the left-hand side. In this paper, for simplicity and reasons of accuracy, we avoid numerical evaluation of $M_{1}$ acting on $\Omega(z)$ when $M_{1}$ is singular. (In earlier work we implemented $M_{1}$ 
numerically $[28,29]$.) This is achieved as follows: the action of $M_{1}$ on the piecewise constant traction $t^{\mathrm{pr}}$ on the righthand side of the equations is evaluated analytically. The action of $M_{1}$ on $\Omega(z)$ on the left-hand side of Eq. (2) followed by application of $P_{j}$, when $\tau$ and $z$ are on the same hole, is evaluated by changing the order of integration

$$
\begin{aligned}
-\mathscr{R} e & \left\{\int_{\Gamma_{j}} \frac{\bar{\tau} \mathrm{d} \tau}{\pi} \int_{\Gamma_{j}} \frac{\Omega(z) \mathrm{d} z}{z-\tau}\right\} \\
= & \mathscr{R} e\left\{\int_{\Gamma_{j}} \frac{\Omega(z) \mathrm{d} z}{\pi} \int_{\Gamma_{j}} \frac{\bar{\tau} \mathrm{d} \tau}{\tau-z}\right\} .
\end{aligned}
$$

The last integral on the right-hand side of Eq. (19) will be evaluated analytically for circular holes. For holes of arbitrary shapes it needs to be evaluated numerically. It is an easier task to evaluate the action of singular integral operators accurately on functions which are known everywhere, than on functions which are known only at a set of nodes.

Remark 4.1. Eq. (1) was originally derived under the assumption of a smooth $\Gamma_{0}$ and for the more regular potential $\phi(z)$, see paragraph 98 of Muskhelishvili [41]. The operator $M_{3}$ is compact under the smoothness assumption. In corners the boundary $\Gamma_{0}$ is merely continuous. As a consequence, the potential $\Phi(z)$ of Eq. (9) has a limiting value on $\Gamma_{0}$ from the inside (the unknown quantity $\Omega(z)$ in Eq. (1)) which in corners may not even be finite. Fortunately, interpreted in a suitably generalized sense, Eq. (1), also holds for domains with corners, see paragraph 99 and 100 of Muskhelishvili [41]. More precisely, the corner points should be excluded from the domain of validity and integrals for which bounded primitive functions exist should be interpreted as generalized Riemann integrals.

\subsection{Quadrature}

This section describes the quadrature used in our discretization scheme. We discuss the quadrature in the context of the integral operator $M_{3}$ acting on $\Omega(z)$. The operator $M_{1}$ poses no extra difficulties, see Section 4.2.

A Nyström scheme means that $\Omega(z)$ is represented by pointwise values on $\Gamma$, and that Eqs. (1) and (2) should be satisfied at those same discretization points. We adopt the term source points for the discretization points where $\Omega(z)$ has support, and the term target points for the discretization points where Eqs. (1) and (2) should be satisfied. Obviously, source points and target points are the same points in a Nyström scheme. Still, it helps to distinguish between the two uses of the points when discussing quadrature techniques. We use the variable $\tau$ when we emphasize the source point aspect, and the variable $z$ when we emphasize the target point aspect of a point. The kernel of $M_{3}$ will be referred to as $M(z, \tau)$.

On regular panels, the density $\Omega(\tau)$ and the kernel $M(z, \tau)$ are smooth functions of $\tau$. They can be well approximated with polynomials. We use 10-point Gauss-Legendre quadrature for the discretization of $M_{3} \Omega(z)$, and we place the discretization points accordingly, see the left image of Fig. 4.

On the corner panels, the density $\Omega(\tau)$ is singular. The nature of $M(z, \tau)$ depends strongly on whether the target point $z$ is on, close to, or far removed from the corner panel. The basic strategy is to switch representation for $\Omega(\tau)$ from $N_{\mathrm{p}}$ complex pointwise values to $2 N_{\mathrm{p}}$ real and imaginary parts of coefficients in expansion (18). Then we evaluate the integrals $M_{3} \phi_{n}(z)$, where $\phi_{n}(\tau)$ are the $2 N_{\mathrm{p}}$ basis functions in Eq. (18). Finally, we multiply the coefficients with the corresponding integrals.

When a target point $z$ is far away from a corner panel and the source points $\tau$ are on a leg of a corner panel, $M(z, \tau)$ is a smooth function of $\tau$. For the evaluation of $M_{3} \phi_{n}(z)$ we use simple 16-point quadrature, based on the values of $M(z, \tau)$ and $\phi_{n}(\tau)$ at 16 temporary points on each corner leg. As for the placement of these temporary points, the GaussLegendre points seems as a reasonable choice.

When target points $z$ are close to, or on, a corner panel and the source points $\tau$ are on a corner panel, $M(z, \tau)$ is not a smooth function of $\tau$. We use adaptive quadrature and evaluate a number of integrals of which we give one particular example. We introduce a parameterization $t$ for

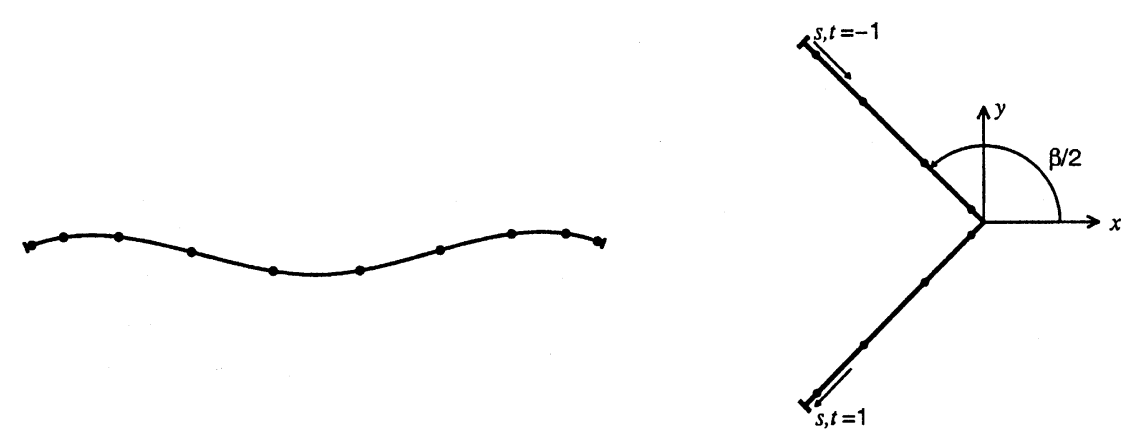

Fig. 4. Left: a regular quadrature panel. Right: a corner quadrature panel with two legs. The dots symbolize points where the solution $\Omega(z)$ has support. 
the local coordinate $\tau_{*}$, and a parameterization $s$ for the local coordinate $z_{*}$, see the right image of Fig. 4. Now $\tau_{*}$ and $z_{*}$ (on the corner panel) range from $\mathrm{e}^{\mathrm{i} \beta / 2}$ to $\mathrm{e}^{-\mathrm{i} \beta / 2}$ as $t$ and $s$ range from -1 to 1 . The action of $M_{3}$ on the basis function $\tau_{*}^{\lambda_{n}-1}$ for $z$ on the first leg can be computed as

$$
\begin{aligned}
& M_{3} \tau_{*}^{\lambda_{n}-1}(z)= \\
& \mathrm{e}^{-\mathrm{i} \beta\left(\lambda_{n}-1\right) / 2}\left[\frac{\left(1-\bar{n}_{z}^{2} n_{\tau}^{2}\right)}{2 \pi \mathrm{i}} \int_{0}^{1} \frac{t^{\lambda_{n}} \mathrm{~d} t}{\left(t^{2}+s^{2}-2 \mathscr{R} e\left\{\bar{n}_{z} n_{\tau}\right\} t s\right)}\right] \\
& \quad+\mathrm{e}^{\mathrm{i} \beta\left(\bar{\lambda}_{n}-1\right) / 2}\left[\frac{\left(1-\bar{n}_{z}^{2} n_{\tau}^{2}\right)}{2 \pi \mathrm{i}} \int_{0}^{1} \frac{t^{\overline{\lambda_{n}}} \mathrm{~d} t}{\left(t-\bar{n}_{z} n_{\tau} s\right)^{2}}\right],
\end{aligned}
$$

$s \leq 0$,

where $n_{z}=\mathrm{i} \mathrm{e}^{\mathrm{i} \beta / 2}$, and $n_{\tau}=-\mathrm{ie}^{-\mathrm{i} \beta / 2}$. Similarly, for a target point $z$ on the second leg we get

$$
\begin{aligned}
& M_{3} \tau_{*}^{\lambda_{n}-1}(z)= \\
& -\mathrm{e}^{\mathrm{i} \beta\left(\lambda_{n}-1\right) / 2}\left[\frac{\left(1-\bar{n}_{z}^{2} n_{\tau}^{2}\right)}{2 \pi \mathrm{i}} \int_{0}^{1} \frac{t^{\lambda_{n}} \mathrm{~d} t}{\left(t^{2}+s^{2}+2 \mathscr{R} e\left\{\bar{n}_{z} n_{\tau}\right\} t s\right)}\right] \\
& -\mathrm{e}^{-\mathrm{i} \beta\left(\bar{\lambda}_{n}-1\right) / 2}\left[\frac{\left(1-\bar{n}_{z}^{2} n_{\tau}^{2}\right)}{2 \pi \mathrm{i}} \int_{0}^{1} \frac{t^{\bar{\lambda}_{n}} \mathrm{~d} t}{\left(t+\bar{n}_{z} n_{\tau} s\right)^{2}}\right], \quad 0 \leq s,
\end{aligned}
$$

where now $n_{z}=-\mathrm{ie}^{-\mathrm{i} \beta / 2}$ and $n_{\tau}=\mathrm{ie}^{\mathrm{i} \beta / 2}$. This example was also given in Ref. [28], along with a more thorough derivation. Unfortunately, there was a misprint in Ref. [28], which is corrected here.

\subsection{Properties of the quadrature}

In Section 4.3 we reviewed the quadrature techniques used. Here we comment on some of the properties of the quadrature and discuss theoretical aspects.

The problem of solving for stress on a multiply connected polygonal domain is inherently well conditioned. With this we mean that a small change in the applied load or in some overall geometric descriptor, such as an included vertex angle, will lead to small changes in most quantities computed from the solution, such as notch stress intensity factors [23] and the pointwise stress-field inside the domain. The problem is, however, ill-conditioned in the sense that a small relative perturbation of a local geometric descriptor, such as the position of a discretization point close to a corner, can lead to a large change in the solution close to that corner. Therefore, even with Fredholm integral equations of the second kind, it is difficult to construct entirely stable algorithms. Great care must be taken to compute geometric quantities as accurately as possible. The dominant source of error, in our algorithm, comes from the corner quadrature. Errors arise, for example, when we invert relation (18). The ill-conditioning of this operation increases with the number of discretization points and terms that are included, and with the spacing of the exponents $\lambda_{n}$ and $\mu_{n}$ of Eqs. (15) and (16). There is, thus, a trade-off between convergence and stability: the more exponents that are included-the more rapid convergence but the lower achievable accuracy in the solution close to corners. In this paper, we have decided to include exponents $\lambda_{n}$ and $\mu_{n}$ which have a real part less than approximately seven. For the problems studied in this paper this means that $N_{\mathrm{p}}$ will range from 8 to 12 .

Let us now consider the placement of the $N_{\mathrm{p}}$ discretization points on a corner panel. For Gaussian quadrature, a correct placement of the nodes ensures the Gaussian property that $2 \mathrm{~N}$ basis functions are integrated exactly. For the quadrature described in Section 4.3, any distinct placement of $N_{\mathrm{p}}$ discretization points on a corner panel will give $2 N_{\mathrm{p}}$ coefficients and enable exact integration of $2 N_{\mathrm{p}}$ basis functions for $\Omega(\tau)$. Unfortunately, we are not interested in merely integrating $\Omega(\tau)$, but the composition $M_{3} \Omega(z)$. For this we need to evaluate $\phi_{n}(\tau)$ at other points than the discretization points, as described in Section 4.3. Anyhow, the placement of the discretization points on a corner panel turns into $N_{\mathrm{p}}$ free parameters which could be chosen as to fine tune the algorithm. One option would be to choose this freedom to minimize the error, in some appropriate norm, of the approximation of $\Omega(\tau)$ with a linear combination of $2 N_{\mathrm{p}}$ basis functions $\phi_{n}(\tau)$. Another option would be to choose the placement of the $N_{\mathrm{p}}$ points as to minimize the condition number of the mapping (18). In this paper, lacking a good theory, we have chosen to place the $N_{\mathrm{p}}$ discretization points symmetrically on the corner panels. On each leg the nodes are placed according to a Gauss-Lobatto quadrature rule for integrals of the type

$\int_{0}^{1} \frac{f(x)}{\sqrt{x}} \mathrm{~d} x$

where $f(x)$ is a smooth function, and the node $x=1$ is fixed.

The determination of which target points $z$ should be considered as being 'far away' from the corner panels must also be made. In the setups studied in this paper the kernel $M(z, \tau)$ is sufficiently smooth as to be accurately evaluated with 16 points on each corner leg when the point $z$ is located a distance further than $4 l_{\mathrm{cp}}$ away from the corner panel.

\subsection{Precomputation and variable precision}

Some quantities needed in the algorithm are precomputed in extended (Fortran REAL $* 16$ and COMPLEX *32) precision. These quantities are then rounded to double (REAL $* 8$ and COMPLEX $* 16$ ) precision and included in the main program. Precomputation of a limited number of quantities is standard in integral equation based solvers [53]. This practice can both save time and increase the stability of the code. Naturally, the more quantities that are precomputed, the more stably the code will run. If we were to precompute all quantities related to corner quadrature in extended precision, our problems with ill-conditioning would go away. This would take time. 
Precomputation in extended precision is equivalent to a special case of variable precision arithmetic where all unknowns and most geometric quantities are declared in double precision, and a few geometric quantities and intermediary variables are declared in extended precision. While, perhaps, unorthodox, variable precision coding could be a simplification over regular precomputation. It eliminates the need for reading and writing on files. The composition of matrices, declared in higher precision, does not need to be carried out explicitly. We believe that variable precision arithmetic could be efficient for particularly difficult problems. Section 5.1 gives a few examples where extended precision is used for the intermediary variables and geometric quantities appearing on the righthand side of Eq. (18). In most numerical examples we use double precision throughout the main program and only precompute, in extended precision, a few quantities such as Gaussian weights and nodes, and inverses of small matrices.

\subsection{Iterative solution and fast multipoles}

We solve the discretized systems (1) and (2) iteratively using the GMRES solver [46]. Compensated summation [31,36] is used whenever deemed beneficial, for example, when computing inner products. The matrix-vector multiplications will be done in a direct way for smaller systems, and with the use of the fast multipole method [5,20, 45] for larger systems. The only part of the action of $M_{3}$ on $\Omega(\tau)$ which cannot be computed with the fast multipole method is the evaluation of $M_{3} \phi_{n}(z)$, when $z$ is close to, or on, a corner panel. The computational work for these integrals scales linearly with the number of corners.

The fast multipole method has previously been used in 2D elastostatic contexts, involving operators similar to $M_{1}$ and $M_{3}$, see Refs. [17-19,26]. The basic strategy is to compute the fields $\Phi(z)$ of Eq. (9) and $\Psi(z)$ of Eq. (10) and then to combine the results as to get $M_{1} \Omega(z)$ and $M_{3} \Omega(z)$. The computation of $M_{3} \Omega(z)$ requires about $70 \%$ more work than the mere computation of $M_{1} \Omega(z)$. In the present code, for simplicity, we use a non-adaptive implementation based on the original scheme of Ref. [20] but which includes some ideas of Ref. [32]. For example, the number of levels of refinement in the hierarchical mesh is not $L=\log _{4} N$, where $N$ is the number of discretization points, but is determined by the condition that no box on the finest mesh level should contain more than $p=-\log _{2}$ tol points, where tol is the requested relative precision. For a uniform distribution of points, this reduces the complexity in precision from $p^{2}$ to $p$, see Fig. 5 and compare Step 1 of Ref. [32]. Also, the costly conversions of multipole expansions into local Taylor

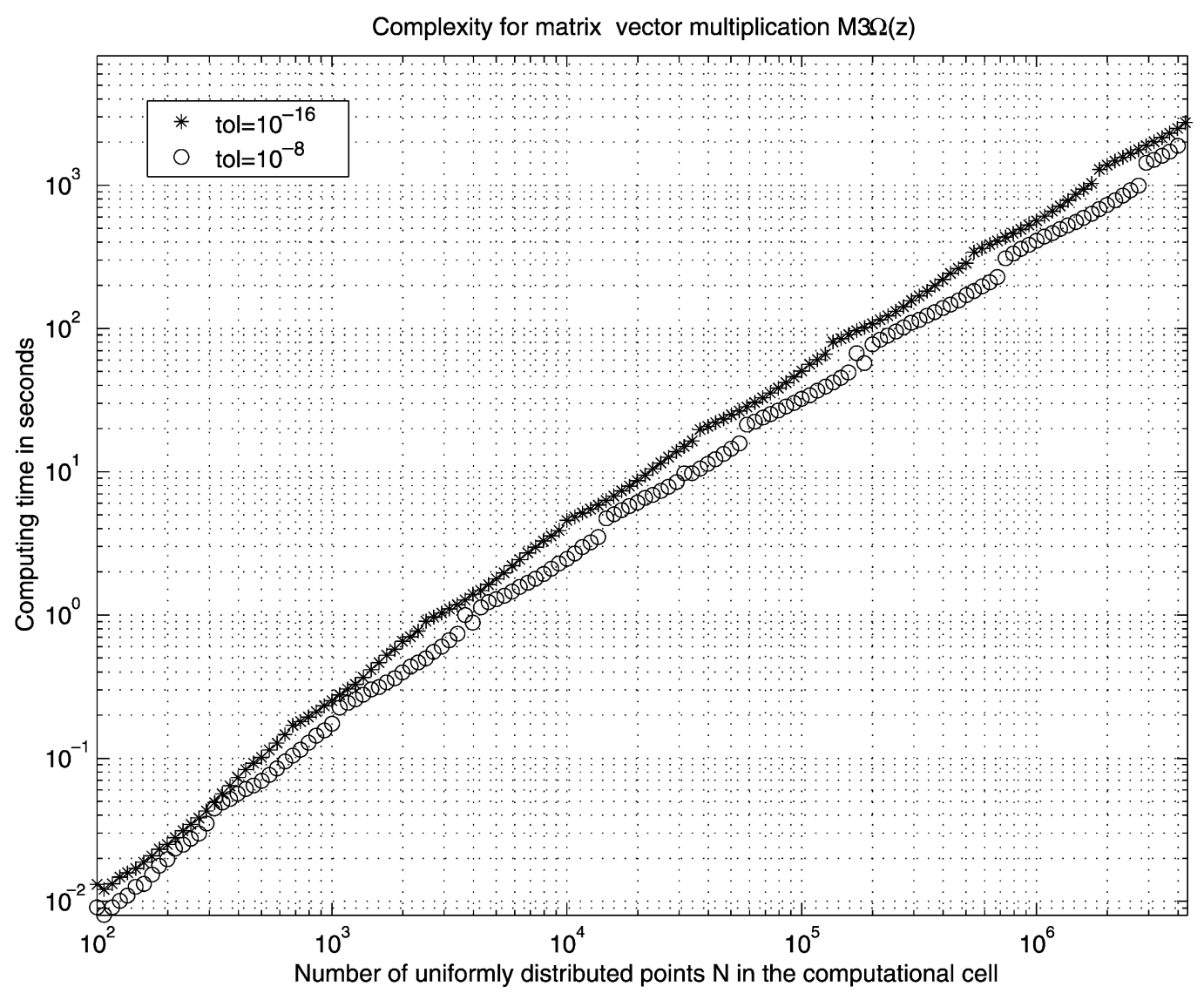

Fig. 5. Computing time on a SunBlade 100 workstation for one matrix-vector multiplication $M_{3} \Omega(z)$ as a function of the number of uniformly (randomly) distributed points $N$ in the computational cell. The field $M_{1} \Omega(z)$ is computed as a partial result in this process. Stars refer to a requested relative precision of $t o l=10^{-16}$ and $p=53$ terms in multipole and local Taylor expansions. Open circles refer to tol $=10^{-8}$ and $p=27$. 
expansions for boxes in the interaction list of a box $b$ are replaced by direct evaluation of multipole fields at points in $b$ if the number of such points is smaller than $p / 2$, compare Step 5 of Ref. [32]. We refrain from giving more details since our code is not entirely optimal with respect to the latest development in the field. More efficient and consequent implementations for some kernels in 2D do exist $[32,15]$. We speculate that speedups with a factor of 5-10 are possible for the problem sizes treated in this paper.

The number of GMRES iterations needed to solve the discretized systems (1) and (2) to a given relative precision in the residual, chiefly depends on the geometry and on the load. It does not depend on the total number of discretization points. This is a consequence of Eqs. (1) and (2) being a Fredholm integral equation of the second kind. The number of GMRES iterations needed does, however, depend mildly on the number of exponents $\lambda_{n}$ and $\mu_{n}$ included in expansion (18). More exponents increase the ill-conditioning of the algorithm, see Section 4.4.

\subsection{Order of accuracy}

In a Nyström scheme, the order of convergence of the unknown quantity is determined by the (worst) quadrature used in the discretization of the integral equation. The order of convergence of post-processed quantities may, of course, also depend on the order of accuracy of the post-processor. In our scheme, the order of convergence for $\Omega(z)$ will, asymptotically, be determined by the magnitude of the smallest exponent $\lambda_{n}$ or $\mu_{n}$ omitted in expansion (18). The notch stress intensity factor $Q_{\mathrm{I}}$ of Eq. (17) is directly computed from $\Omega(z)$ in the corners and will have the same order of convergence. The stress field inside $D$ is computed as integrals over $\Omega(z)$. The asymptotic order of convergence for the stress field at a given point in $D$ will be of the order one higher than for $Q_{\mathrm{I}}$ since the quadrature has higher order on regular panels than on corner panels, and since the relative number of corner panels decreases as the mesh is refined. The same holds true for the stress concentration factor $K_{\mathrm{t}}$ of Eq. (12). We compute $K_{\mathrm{t}}$ in a post-processor using Newton's method, safeguarded with Golden Section Search. This procedure is, in itself, 9th order accurate.

\section{Numerical examples}

This section presents numerical examples. We start with some simple standard setups in order to verify the properties of our algorithm, stated in Section 4, and to compare with previous results. We end with a rather complex, large-scale computation.

\subsection{Some SENT specimens}

The notch stress intensity factor $Q_{\mathrm{I}}$ of Eq. (13) of a single edge notched specimen under uniaxial tension (SENT), such as the one in Fig. 2, has been computed by numerous researchers during the last 30 years. A common setup has a height to width ratio of $h / w=1$, a relative notch depth of $a / w=0.5$ and an included vertex angle of $\beta=3 \pi / 2$. A typical agreement between the results of different authors is three digits, see Ref. [28], Table 1. The most accurate computation, $Q_{\mathrm{I}}=4.2959$, was done by Strandberg [50].

We performed four sets of computations, presented in Fig. 6. First we used $N_{\mathrm{p}}=8$ points on all corner panels. The smallest exponent $\lambda_{n}$ or $\mu_{n}$ not fully included in the expansion of Eq. (18) was $\lambda_{5}$ in the corner $E$ of Fig. 2. The real part of this exponent is approximately 5.65, which corresponds well with the slope of the line formed by the open circles in Fig. 6. Next, we increased the number of points on the corner panel at corner $E$ to $N_{\mathrm{p}}=10$, while all other corner panels still have $N_{\mathrm{p}}=8$. The smallest exponent not fully included in the expansion of Eq. (18) is now $\lambda_{6}$ in the corner $E$. The real part of this exponent is approximately 6.98. The stars in Fig. 6 show that the convergence now is so rapid that we do not reach the asymptotic regime, in IEEE DP arithmetic, until the instabilities in the algorithm start to pollute the result. Therefore, we also performed variable precision arithmetic computations, see Section 4.5, and full quadruple precision computation, shown as triangles and diamonds in Fig. 6, respectively. The asymptotic regime is reached at about 3000 discretization points and at a relative precision in $Q_{\mathrm{I}}$ of about $10^{-16}$. The number of GMRES iterations needed in all these computations was around 40.

We also include results for some SENT specimens with a larger included vertex angle $\beta$. These geometries are considered more difficult to treat [50]. The largest angle for which we have found computations in the literature is $\beta=11 \pi / 6$. We solve for a few setups with $\beta=11 \pi / 6$ using a number of $N_{\mathrm{p}}=12$ points on the corner panel containing the notch, and compare with previous results. Gross and Mendelson [23] report a value of $Q_{\mathrm{I}}=3.569$ for a setup with $a / w=0.5$ and $h / w=1.2$. At about 1600 discretization points our computations converge to a value of $Q_{\mathrm{I}}=$ 3.5696698583(19), where the two digits within parenthesis are obtained using variable precision arithmetic. Strandberg [50] report a value of $Q_{\mathrm{I}}=3.5696$ for a setup with $a / w=$ 0.5 and $h / w=2$. At about 2300 discretization points our computations converge to a value of $Q_{\mathrm{I}}=$ 3.5696265724(35). Chen [11] report a value of $Q_{\mathrm{I}}=$ 3.568 for a setup with $a / w=0.5$ and $h / w=3$. At about 3000 discretization points our computations converge to a value of $Q_{\mathrm{I}}=3.5696260183(43)$. The number of GMRES iterations needed in all these computations was around 60.

\subsection{A square with one hole}

A very simple finite and multiply connected domain is the unit square with a circular hole of radius $R$ centered at the origin. The problem of determining the stress concentration factor $K_{\mathrm{t}}$ of Eq. (12) for the hole, when a uniaxial stress is applied, has been treated by Isida and Sato [33] and 


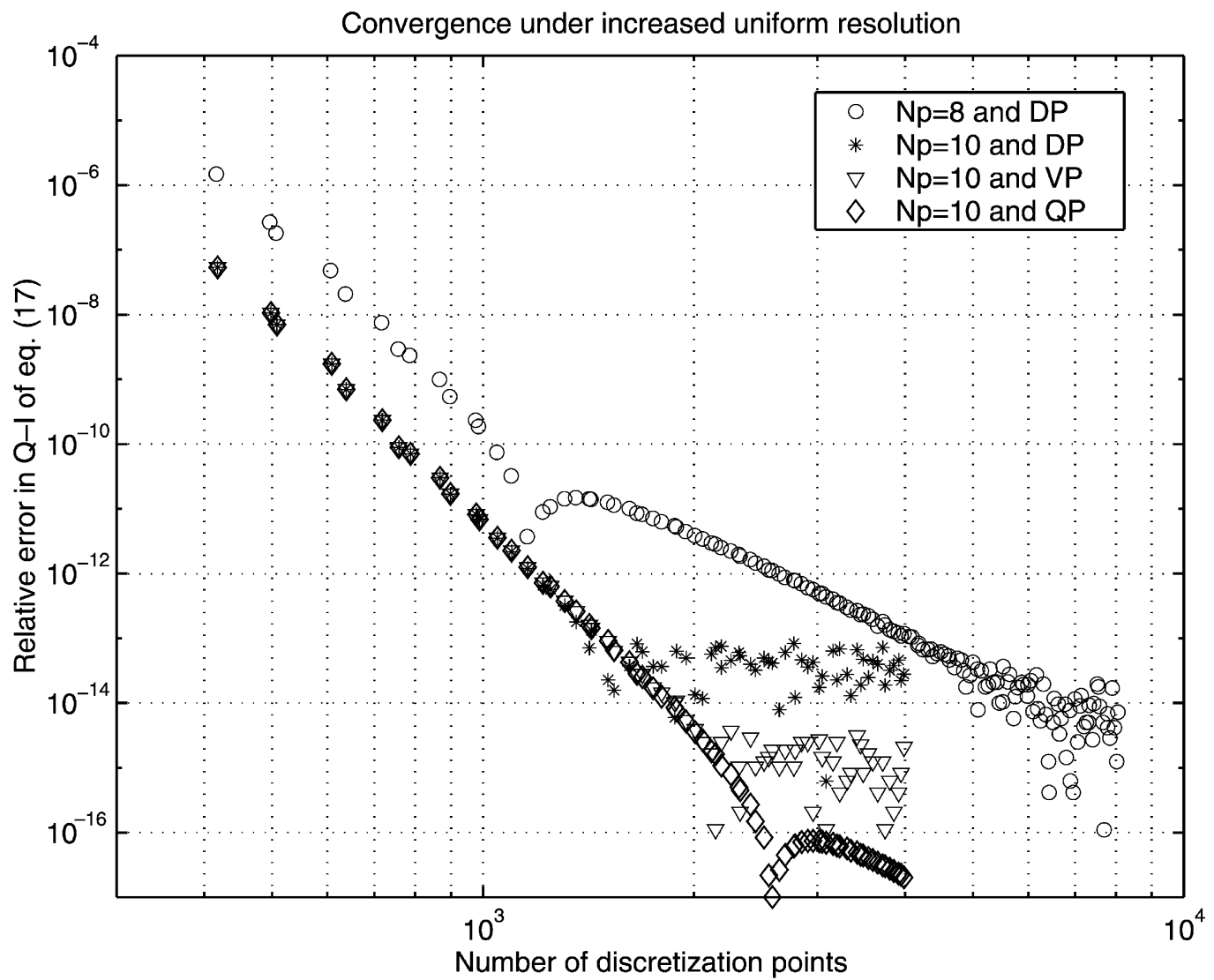

Fig. 6. Convergence of the notch stress intensity factor $Q_{\mathrm{I}}$ of Eq. (17) for the setup in Fig. 2. The geometry is chosen so that $h / w=1, a / w=0.5$ and $\beta=3 \pi / 2$. The reference value is chosen as $Q_{\mathrm{I}}=4.2958869676986369$. DP refers to double precision arithmetic, VP to variable precision arithmetic, and QP to quadruple precision arithmetic. $N_{\mathrm{p}}$ is the number of discretization points on the corner panel containing the notch.
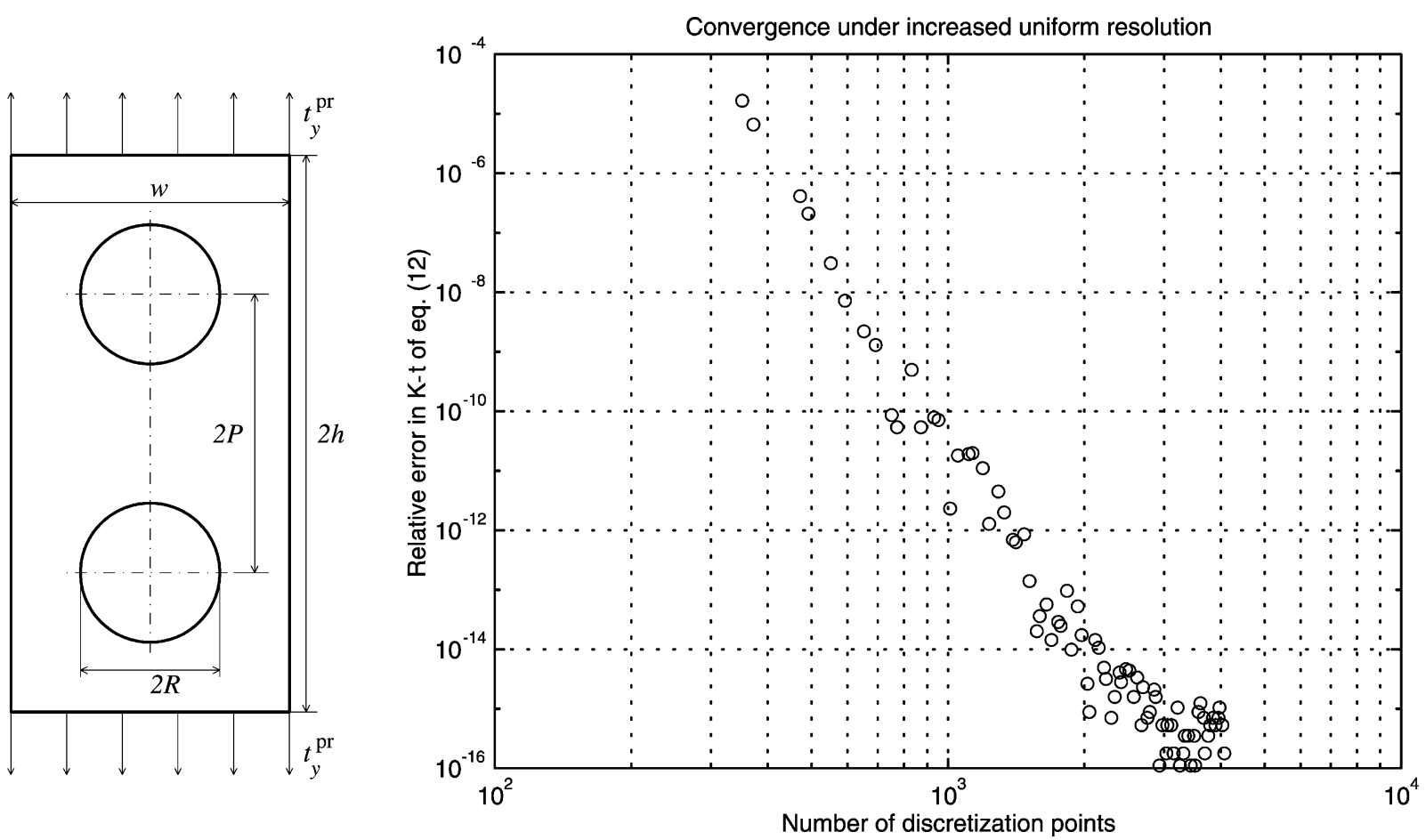

Fig. 7. Left: two symmetrically placed holes of radius $R$ separated a distance $2 P$ in a rectangular plate with height $2 h$ and width $w$. Uniaxial stress $t_{y}^{\mathrm{pr}}$ is prescribed at two opposing sides. Right: convergence of the stress concentration factor $K_{\mathrm{t}}$ of Eq. (12). The geometry is chosen so that $h / w=1,2 P=w$ and $R=w / 4$. The reference value is chosen as $K_{\mathrm{t}}=5.0438755094142715$. The computations are done in double precision arithmetic. 


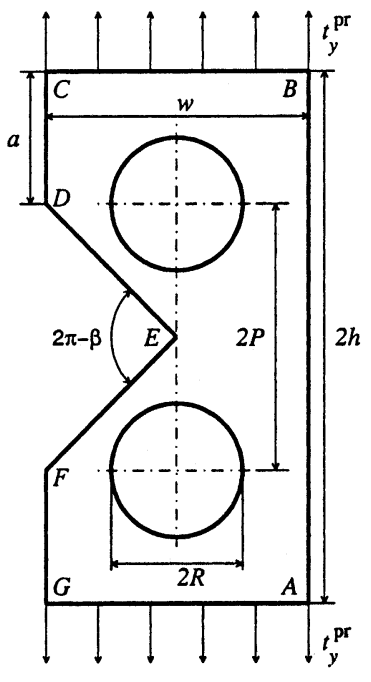

Fig. 8. Two symmetrically placed holes of radius $R$ separated a distance $2 P$ in a single edge notched specimen with height $2 h$ and width $w$. The notch depth is $a$. The included vertex angle is $\beta$. Uniaxial stress $t_{y}^{\mathrm{pr}}$ is prescribed at two opposing sides.

by Nisitani and Chen [42]. For a hole with radius $R=0.25$ they report the values $K_{\mathrm{t}}=6.3887$ and 6.38869 , respectively. Our computations reproduce their values. With 2000 discretization points we get convergence to $K_{\mathrm{t}}=$ 6.3886960194568 .

\subsection{A rectangle with two holes}

A rectangle with two holes is depicted in the left image of Fig. 7. We choose the same height to width ratio as for the first SENT specimen in Section 5.1, that is, $h / w=1$. We take the separation distance to be $2 P=w$ and the radius to be $R=w / 4$. Double precision arithmetic is used throughout the code. A convergence study for the stress concentration factor $K_{\mathrm{t}}$ of Eq. (12) is presented in the right image of Fig. 7.

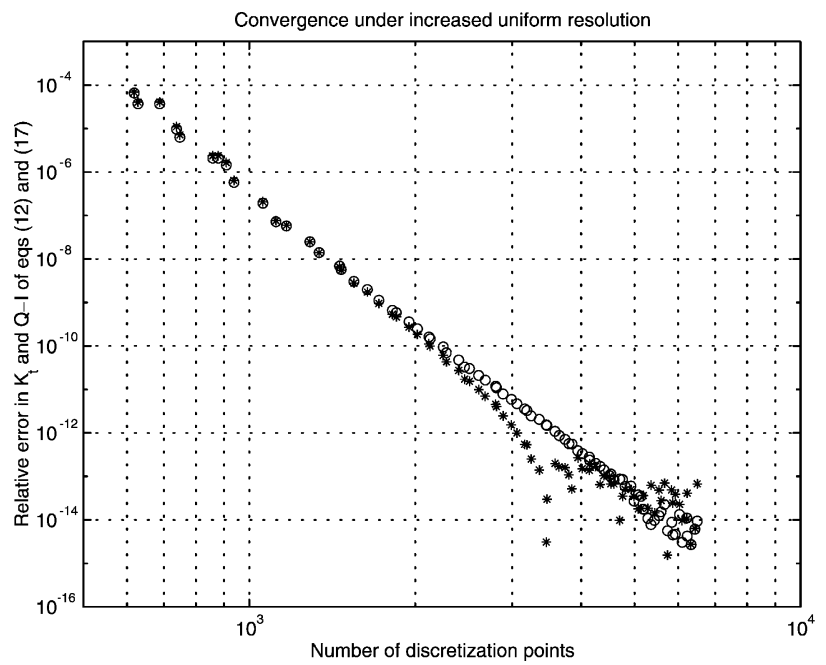

Note that the problem is well-conditioned and that we do not reach the asymptotic regime: the convergence rate is dominated by the 9th order error in the optimization process, see Section 4.7.

\subsection{A SENT specimen with two holes}

We now combine the geometries of Figs. 2 and 7, left image, to get the perforated SENT specimen of Fig. 8. As before, we take $h / w=1,2 P=w, R=w / 4, a / w=0.5$ and $\beta=3 \pi / 2$. We perform one set of computations for $Q_{\mathrm{I}}$ and $K_{\mathrm{t}}$ where the matrix-vector multiplications are done in a direct way, see the left image of Fig. 9, and one set of computations where the matrix-vector multiplications are accelerated with the fast multipole method, see the right image of Fig. 9. Note that the direct computations give a couple of extra correct digits. The direct computations, involving the actual construction of the discretized matrix $M_{3}$, converge better than the multipole accelerated computations since we have taken advantage of that some of the entries in $M_{3}$ can be expressed on analytic form, and since we used compensated summation for the direct matrixvector multiplications.

\subsection{A SENT specimen with 1170 holes}

Lastly, in a large-scale example, we increase the number of holes in the SENT specimen of Fig. 8 substantially. Again we take $h / w=1, a / w=0.5$ and $\beta=$ $3 \pi / 2$. We then construct a square grid on the specimen. The origin is at the notch-tip $E$. The grid points are separated a distance $d$ from their nearest neighbors and placed at coordinates given by $x=0.5 d+i d$ and $x=$ $0.5 d+j d$ where $i$ and $j$ are integers. The separation distance $d$ is given by $d=w / n$ where $n$ is an even integer. Grid points on the boundary are removed. Now

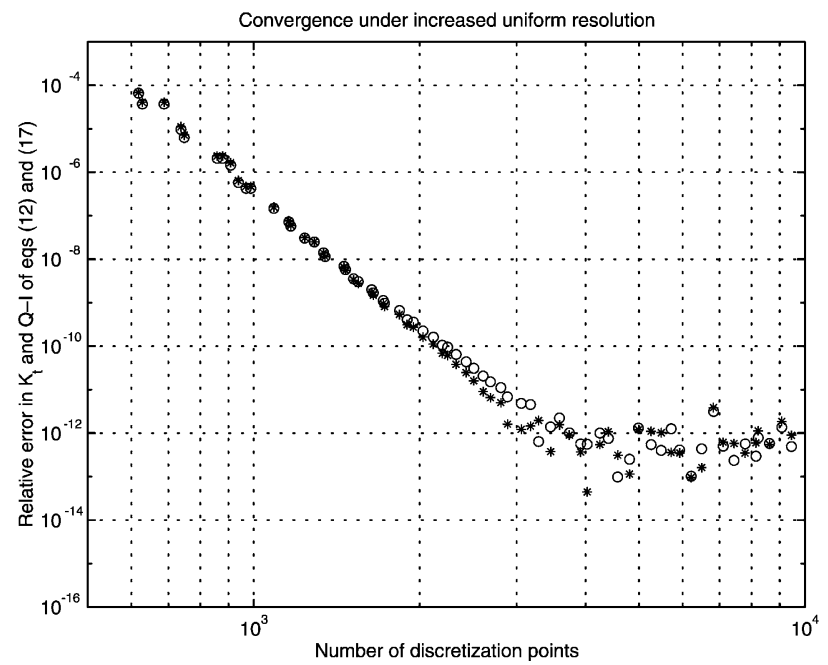

Fig. 9. Convergence of the notch stress intensity factor $Q_{\mathrm{I}}$ of Eq. (17), shown as stars, and the stress concentration factor $K_{\mathrm{t}}$ of Eq. (12), shown as open circles, for the setup in Fig. 8. Left: matrix-vector multiplications are computed in a direct manner. Right: matrix-vector multiplications are accelerated with the fast multipole method. Reference values are taken as $Q_{\mathrm{I}}=5.1691735817822$ and $K_{\mathrm{t}}=15.10033207977245$. 


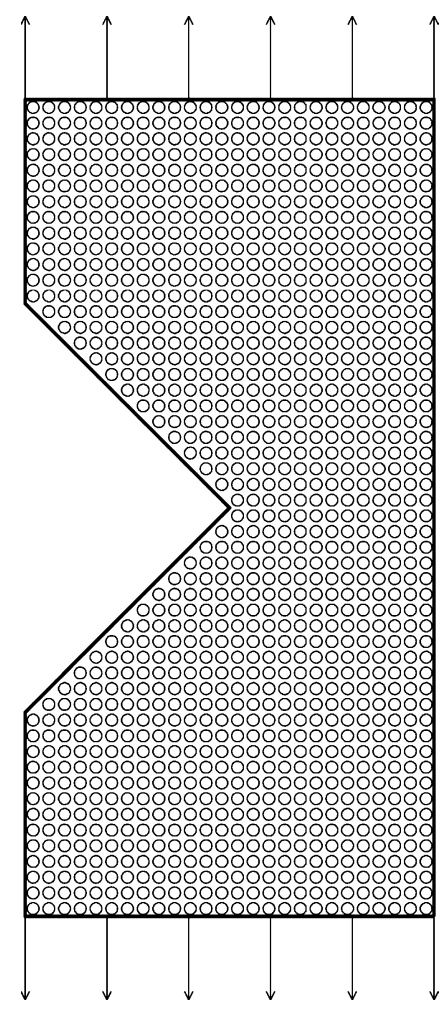

Fig. 10. A number of 1170 equisized circular holes in the SENT specimen of Fig. 2. The holes are placed on a square grid. The radii of the holes are $3 / 8$ of their separation distance. Uniaxial stress $t_{y}^{\mathrm{pr}}$ is prescribed at two opposing sides.

we place a circular hole with radius $R=3 d / 8$ at each grid point. There will be $7 n^{2} / 4-n / 2$ such holes. We choose $n=26$ and get 1170 holes, see Fig. 10. A convergence study of the quantities $Q_{\mathrm{I}}$ and $K_{\mathrm{t}}$ is given in the left image of Fig. 11. We refined the mesh until our workstation ran out of memory. The number of GMRES iterations needed for full convergence was about 110 and

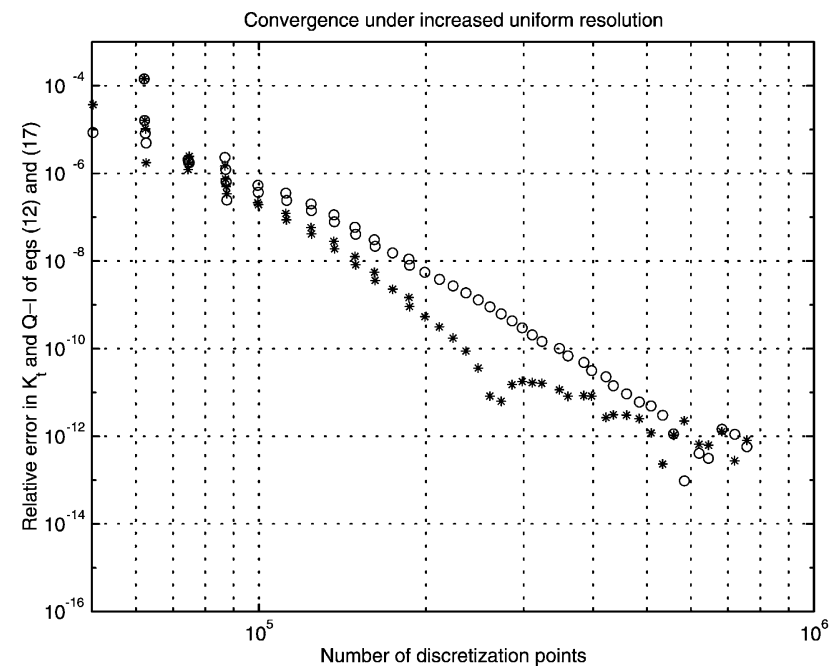

independent of the number of discretization points. See the right image of Fig. 11 for an iteration history.

One could expect that the achievable accuracy of $Q_{\mathrm{I}}$ and $K_{\mathrm{t}}$ should be lower in the present large-scale example than in the small-scale example of Section 5.4. After all, a hundred times more discretization points and closer spacing between the points on the boundary would lead to larger errors. One can see, by comparing the left image of Fig. 11 with the right image of Fig. 9, that the convergence in this large-scale example is almost as stable as the convergence in the much smaller example. Our explanation for this is that both the notch and the hole, for which the maximum in Eq. (12) occurs, are located close to the origin where the relative accuracy of computed positions is higher than elsewhere in the computational cell.

\section{Conclusions and discussion}

This paper presents numerical results for stress computations on multiply connected finite polygonal domains. The algorithm is based on the second kind Fredholm integral Eqs. (1) and (2) which are discretized using a Nyström scheme and composite quadrature. The analytic properties of the unknown quantity enable the use of high order quadrature. Pointwise representation enables easy incorporation of the fast multipole method into an iterative solver. Computations of notch stress intensity factors and stress concentration factors are obtained from the solution via simple post-processors. Convergence to high accuracy was demonstrated for a SENT perforated with 1170 circular holes.

Eqs. (1) and (2) hold for arbitrarily shaped domains with applied external traction. Our algorithm specializes to polygonal domains, circular holes, and piecewise constant traction. The reasons for restricting our focus are that these

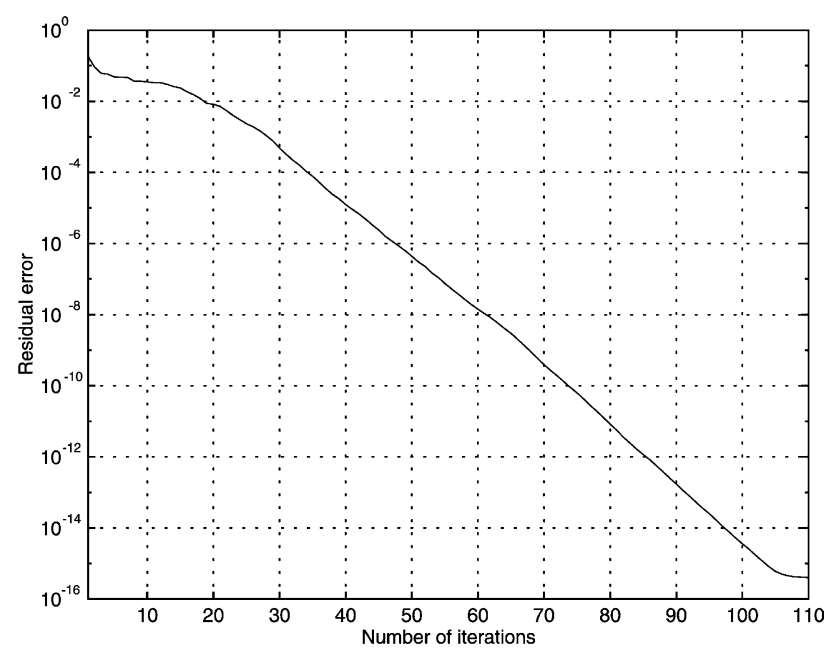

Fig. 11. Left: convergence of the notch stress intensity factor $Q_{\mathrm{I}}$ of Eq. (17), shown as stars, and the stress concentration factor $K_{\mathrm{t}}$ of Eq. (12), shown as open circles, for the setup in Fig. 10. Reference values are taken as $Q_{\mathrm{I}}=11.02821540165$ and $K_{\mathrm{t}}=55.06854533610$. Right: a GMRES iteration history for a computation with $N=756,938$ discretization points. 
geometries are common in the engineering literature and that we want to establish benchmarks for simply reproducible setups. A more general solver would require more work, as we now outline: allowing for non-circular holes and for nonconstant tractions is simple. The circular property of the holes is used in the analytic evaluation of the rightmost integral of Eq. (19). This integral only involves known geometric quantities and it can be evaluated numerically almost without precision loss for holes of arbitrary shapes. The situation is similar for the piecewise constant property of the applied traction, which is used for analytic evaluation of the right-hand sides of Eqs. (1) and (2). In Ref. [27] we have successfully tested a numerical implementation of the operator $M_{1}$ in this context. Allowing for curved corner boundaries is harder. Far away from corners, no assumption about the shape of the boundary is made. Close to, or on, corner panels, however, we use the property that the corner legs are straight-both in the analysis of the stress field leading up to the expansion (14) and in the evaluation of the action on the operator $M_{3}$ on the basis functions in this series. The expansion (14) will hold, asymptotically, also for curved corner boundaries, but the extraction of the expansion coefficients will become more difficult in the absence of symmetry. Furthermore, the integrals corresponding to those in Eqs. (20) and (21) will be more involved and we can make less use of precomputation when corner panels of different sizes are no longer congruent. These modifications are likely to degrade the performance of the code and may force us to lower the order of the scheme. Yet another extension is to allow for displacement boundary conditions. This requires the replacement of the Eqs. (1) and (2), based on the potentials $\Phi(z)$ and $\Psi(z)$, with equally efficient Fredholm equations for the displacement problem. This problem is open and its solution could involve an analysis resembling that of Ref. [29] but based on the potentials $\phi(z)$ and $\psi(z)$, which are primitive functions of $\Phi(z)$ and $\Psi(z)$. See also Sherman [47].

Disregarding the present restrictions in applicability, our algorithm is still not entirely ideal. Its performance degrades for large included vertex angles. The use of a Galerkin scheme, instead of a Nyström scheme, could, perhaps, eliminate some ill-conditioning related to the change of representation on corner panels. Another worry is that a number of parameters related to the quadrature on the corner panels are determined in an ad hoc manner. More optimal, and theoretically derived, choices could make the scheme even more efficient. If an effort in this direction is worthwhile or not depends on the need for better stability and on the progress of alternative approaches. Work by Yarvin and Rokhlin, involving generalized Gaussian quadrature and singular value decomposition of integral operators on corners, is reported to be in preparation [53]. A more radical way to go is to abandon the idea of solving integral equations completely, and instead compute the solution to the stress problem directly, as a sum of reflected layer potentials. Promising work on this type of fast direct solvers have been reported by Ethridge and Greengard for Laplace's and Poisson's equations on the unit square, and extensions to complex geometry are underway [15].

We have avoided giving explicit timings for our computations. The reason is that we focused more on testing the stability of our scheme than on achieving the fastest possible execution. All tolerances, for example in the fast multipole method and in the GMRES iterative solver, have been set to approximately machine epsilon, corresponding to $p=53$ terms in multipole and local Taylor expansions. Compensated summation has been used in all direct matrix-vector multiplications. As for the large-scale example of Section 5.5, we can say the following: virtually, all computational work takes place in the fast multipole subroutine. The largest computation that we undertook, with 756,938 discretization points and 110 iterations in Section 5.5 , required approximately $18 \mathrm{~h}$ of computing time on a SunBlade 100 workstation using the compiler option (-O4).

\section{Acknowledgements}

This work was supported by NFR, TFR, and The Knut and Alice Wallenberg Foundation under TFR contract 99380 and by the Swedish Research Science Council under contract 621-2001-2799.

\section{References}

[1] Ainsworth M, Guo B. An additive Schwarz preconditioner for $p$ version boundary element approximation of the hypersingular operator in three dimensions. Numer Math 2000;85(3):343-66.

[2] Atkinson C, Bastero JM, Martínez-Esnaola JM. Stress analysis in sharp angular notches using auxiliary fields. Engng Fract Mech 1988; 31(4):637-46.

[3] Atkinson KE. The numerical solution of integral equations of the second kind. Cambridge: Cambridge University Press; 1997.

[4] Bramble JH, Pasciak JE, Schatz AH. The construction of preconditioners for elliptic problems by substructuring. I. Math Comput 1986; 47(175):103-34.

[5] Carrier J, Greengard L, Rokhlin V. A fast adaptive multipole algorithm for particle simulations. SIAM J Sci Stat Comput 1988; 9(4):669-86.

[6] Chan RH, Sun H-W, Ng W-F. Circulant preconditioners for illconditioned boundary integral equations from potential equations. Int J Numer Meth Engng 1998;43(8):1505-21.

[7] Chau KT, Wang YB. A new boundary integral formulation for plane elastic bodies containing cracks and holes. Int J Solids Struct 1999; 36(14):2041-74.

[8] Chen JT, Hong H-K. Review of dual boundary element methods with emphasis on hypersingular integrals and divergent series. Appl Mech Rev 1999;52(1):17-32.

[9] Chen KT, Ting K, Yang WS. Stress analysis of two-dimensional perforated plates using boundary element alternating method. Comput Struct 2000;75(5):515-27.

[10] Chen CS, Krause R, Pettit RG, Banks-Sills L, Ingraffea AR. Numerical assessment of $T$-stress computation using a $p$-version finite element method. Int J Fract 2001;107(2):177-99.

[11] Chen DH. Stress intensity factors for V-notched strip under tension or in-plane bending. Int J Fract 1994;70(1):81-97. 
[12] Cheng H, Rokhlin V, Yarvin N. Nonlinear optimization, quadrature, and interpolation. SIAM J Optim 1999;9(4):901-23.

[13] Cheng H, Greengard L, Rokhlin V. A fast adaptive multipole algorithm in three dimensions. J Comput Phys 1999;155(2):468-98.

[14] Daux C, Moës N, Dolbow J, Sukumar N, Belytschko T. Arbitrary branched and intersecting cracks with the extended finite element method. Int J Numer Meth Engng 2000;48(12):1741-60.

[15] Ethridge F, Greengard L. A new fast-multipole accelerated Poisson solver in two dimensions. SIAM J Sci Comput 2001;23(3):741-60.

[16] Fu Y, Klimowski KJ, Rodin GJ, Berger E, Browne JC, Singer JK, Van de Geijn RA, Vemaganti KS. A fast solution method for 3D manyparticle problems of linear elasticity. Int J Numer Meth Engng 1998; 42(7):1215-29.

[17] Greenbaum A, Greengard L, Mayo A. On the numerical solution of the biharmonic equation in the plane. Physica D 1992;60(1-4): $216-25$.

[18] Greengard L, Helsing J. On the numerical evaluation of elastostatic fields in locally isotropic two-dimensional composites. J Mech Phys Solids 1998;46(8):1441-62

[19] Greengard L, Kropinski MC, Mayo A. Integral equation methods for Stokes flow and isotropic elasticity in the plane. J Comput Phys 1996; 125(2):403-14

[20] Greengard L, Rokhlin V. A fast algorithm for particle simulations. J Comput Phys 1987;73(2):325-48.

[21] Greengard L, Rokhlin V. A new version of the fast multipole method for the Laplace equation in three dimensions. Acta Numerica 1997, Cambridge: Cambridge University Press; 1997. p. 229-69.

[22] Greengard L, Wandzura S. Fast multipole methods. IEEE Comput Sci Engng 1998;5(3):16-56.

[23] Gross B, Mendelson A. Plane elastostatic analysis of V-notched plates. Int J Fract Mech 1972;8:267-76.

[24] Hackbusch W. Multi-grid methods and applications. New York: Springer; 1985.

[25] Hackbusch W. Integral equations. Basel: Birkhäuser; 1995.

[26] Helsing J. Fast and accurate numerical solution to an elastostatic problem involving ten thousand randomly oriented cracks. Int J Fract 1999;100(4):321-7.

[27] Helsing J, Jonsson A. A seventh order accurate and stable algorithm for the computation of stress inside cracked rectangular domains, Department of Solid Mechanics KTH research report TRITA-HLF294; 2001

[28] Helsing J, Jonsson A. On the computation of stress fields on polygonal domains with V-notches. Int J Numer Meth Engng 2002;53(2): 433-53.

[29] Helsing J, Jonsson A. Stress calculations on multiply connected domains. J Comput Phys 2002;176(2):456-82.

[30] Her SH. Fracture analysis of interfacial crack by global-local finite element. Int J Fract 2000;106(2):177-93.

[31] Higham NJ. Accuracy and stability of numerical algorithms. Philadelphia: SIAM; 1996. p. 92-7.

[32] Hrycak T, Rokhlin V. An improved fast multipole algorithm for potential fields. SIAM J Sci Comput 1998;19(6):1804-26.

[33] Isida M, Sato R. Analysis of finite regions containing a circular hole or inclusion with application to their periodic arrays. Trans Jpn Soc Mech Engrs A 1984;50:1619-27.
[34] Jaswon MA, Symm GT. Integral equation methods in potential theory and elastostatics. London: Academic Press; 1977.

[35] Jou HJ, Leo PH, Lowengrub JS. Microstructural evolution in inhomogeneous elastic media. J Comput Phys 1997;131(1):109-48.

[36] Kahan W. Further remarks on reducing truncation errors. Commun Assoc Comput Machinery 1965;8:40.

[37] Linkov AM, Mogilevskaya SG. Complex hypersingular integrals and integral equations in plane elasticity. Acta Mech 1994;105(1-4): 189-205.

[38] Liu YJ, Xu N, Luo JF. Modeling of interphases in fiber-reinforced composites under transverse loading using the boundary element method. ASME J Appl Mech 2000;67(1):41-9.

[39] McLean W. Strongly elliptic systems and boundary integral equations. New York: Cambridge University Press; 2000.

[40] Mikhlin SG. Integral equations. London: Pergamon Press; 1957.

[41] Muskhelishvili NI. Some basic problems of the mathematical theory of elasticity. Groningen: P. Noordhoff Ltd; 1953.

[42] Nisitani H, Chen DH. Body force method and its applications to numerical and theoretical problems in fracture and damage. Comput Mech 1997;19(6):470-80.

[43] von Petersdorff T, Stephan EP. On the convergence of the multigrid method for a hypersingular integral equation of the first kind. Numer Math 1990;57(4):379-91.

[44] Portela A, Aliabadi MH. The dual boundary element method: effective implementation for crack problems. Int J Numer Meth Engng 1992;33(6):1269-87.

[45] Rokhlin V. Rapid solution of integral equations of classical potential theory. J Comput Phys 1985;60:187-207.

[46] Saad Y, Schultz MH. GMRES: a generalized minimum residual algorithm for solving nonsymmetric linear systems. SIAM J Sci Stat Comput 1986;7(3):856-69.

[47] Sherman DI. On the problem of plane strain in non-homogeneous media. In: Olszak W, editor. Non-homogeneity in elasticity and plasticity. London: Pergamon Press; 1959. p. 3-20.

[48] Sokolnikoff IS. Mathematical theory of elasticity. New York: McGraw-Hill; 1956.

[49] Steinbach O, Wendland WL. The construction of some efficient preconditioners in the boundary element method. Adv Comput Math 1998;9(1-2):191-216.

[50] Strandberg M. A numerical study of the elastic stress field arising from sharp and blunt V-notches in a SENT-specimen. Int J Fract 2000; 100(4):329-42.

[51] Williams ML. Stress singularities resulting from various boundary conditions in angular corners of plates in extension. ASME J Appl Mech 1952;19:526-8

[52] Xiao QZ, Karilahoo BL, Williams FW. Application of penaltyequilibrium hybrid stress element method to crack problems. Engng Fract Mech 1999;63(1):1-22.

[53] Yarvin N, Rokhlin V. Generalized Gaussian quadratures and singular value decompositions of integral operators. SIAM J Sci Comput 1998; 20(2):699-718.

[54] Yoshida KI, Nishimura N, Kobayashi S. Application of fast multipole Galerkin boundary integral equation method to elastostatic crack problems in 3D. Int J Numer Meth Engng 2001;50(3):525-47. 